\title{
Adición de botellas plásticas pet en la elaboración de bloques de adobe para viviendas unifamiliares y su efecto en la variación de temperatura y acondicionamiento acústico en el cantón Ambato, provincia de Tungurahua.
}

Addition of pet plastic bottles in the manufacture of adobe blocks for singlefamily homes and his effect on the variation of temperature and acoustic conditioning in the Ambato city, Tungurahua estate.

Galo Wilfrido Núñez Aldás. ${ }^{1}$, Alex Gustavo López Arboleda. ${ }^{2}$, Diego Sebastián Chérrez Gavilanes. ${ }^{3}$ \& Jorge Javier Guevara Robalino. ${ }^{4}$

\begin{abstract}
.
DOI: https://doi.org/10.33262/cienciadigital.v5i1.1536

Introduction. Thermal and acoustic comfort in side of the home room's are important characteristics to reduce the use of electrical devices used for these purposes and consequently the consumption of electrical energy or fuels, this type of savings in homes is important to reduce your expenses of basic services. With this they can increase their economic level. Objective. Analyze the results of the study of the variation of the temperature and acoustic conditioning in adobe blocks, adding plastic recycled type PET. Methodology. The research design was experimental, quantitative and qualitative. The adobe blocks were made with the Peruvian standard E.080. adding crushed PET plastic depending on the volume of the soil used being: $0 \%, 5 \%, 10 \%$ and $15 \%$. For the analysis of temperature and sound, it was based on Chilean standards, using an airtight chamber. Results. Among the results frequently obtained are when

\footnotetext{
${ }^{1}$ Universidad Técnica de Ambato, Facultad de Ingeniería Civil y Mecánica, Carrera de Ingeniería Civil. Ambato, Ecuador.gw.nunez@uta.edu.ec, https://orcid.org/0000-0001-7087-1213

${ }^{2}$ Universidad Técnica de Ambato, Facultad de Ingeniería Civil y Mecánica, Carrera de Ingeniería Civil. Ambato, Ecuador. ag.lopez@uta.edu.ec, https://orcid.org/0000-0001-9914-4925

${ }^{3}$ Universidad Técnica de Ambato, Facultad de Ingeniería Civil y Mecánica, Carrera de Ingeniería Civil. Ambato, Ecuador. ds.cherrez@uta.edu.ec, https://orcid.org/0000-0001-9614-900X

${ }^{4}$ Universidad Técnica de Ambato, Facultad de Ingeniería Civil y Mecánica, Carrera de Ingeniería Civil. Ambato, Ecuador. jj.guevara@uta.edu.ec, https://orcid.org/0000-0002-8314-6712
} 
the Percentage of PET is increased, the insulation is increased by external temperature, giving an ambient temperature of $15^{\circ} \mathrm{C}$, with regard to the sound part, an increase in the insulation level of a $50.97 \%$ of the maximum level emitted by the source. Conclusion. The addition of materials such as PET plastic increases the acoustic and thermal insulation properties of adobe, but always maintaining a relationship with which its minimum resistance does not decrease.

Keywords: Adobe, plastic PET, isolation, acoustic, thermic.

\section{Resumen.}

Introducción. El confort térmico y acústico dentro de las habitaciones de una vivienda constituyen características importantes para reducir la utilización de electrodomésticos empleados para estos fines y en consecuencia el consumo de energía eléctrica o combustibles, este tipo de ahorro en los hogares es importante para reducir sus gastos de servicios básicos y con esto puedan mejorar su nivel económico. Objetivo. Analizar los resultados del estudio de la variación de la temperatura y acondicionamiento acústico en bloques de adobe, adicionando plástico triturado tipo PET. Metodología. El diseño de investigación fue experimental, cuantitativa y cualitativa. La elaboración de bloques de adobe se efectuó con la norma peruana E.080. adicionando plástico triturado PET en función de volumen del suelo utilizado siendo: $0 \%, 5 \%, 10 \%$ y $15 \%$. Para el análisis de temperatura y sonido, se basó en las normas chilenas, empleando una cámara hermética. Resultados. Entre los resultados obtenidos con frecuencia son cuando se aumenta el Porcentaje de PET se incrementa la aislación por temperatura externa, dando una temperatura ambiente de $15^{\circ} \mathrm{C}$, en lo referente a la parte sonora también se obtiene un aumento en el nivel de aislamiento de un $50.97 \%$ del nivel máximo emitido por la fuente. Conclusión. La adición de materiales como el plástico PET, incrementa las propiedades de aislamiento acústico y térmico del adobe, pero siempre manteniendo una relación con la cual no disminuya su resistencia mínima.

Palabras claves: Adobe, plástico PET, aislamiento, acústico, térmico.

\section{Introducción.}

La problemática que ha generado el impacto ambiental alrededor del mundo hace que la humanidad busque alternativas para aprovechar de forma adecuada los recursos que ofrece la naturaleza. (Ordoñez \& Roda, 2016). Desde el inicio de la humanidad el ser humano se ha caracterizado por estar continuamente probando distintos materiales, procesos o métodos constructivos que le sirvan para afrontar los riesgos y factores externos que puedan aparecer. (Muentes, 2016). El suelo (adobe) es uno de los principales recursos naturales que se ha venido utilizando para este fin, y tiene su origen en el descubrimiento de que, al humedecerse, la tierra que contiene arcilla es plástica y fácil de moldear. Luego al secarse se endurece y puede alcanzar una resistencia 
importante a compresión. (Llumitasig "Siza, 2017).

La falta de investigación científico-técnica para implementar al adobe en la construcción carece de opciones valederas, por lo cual es preciso poner mayor énfasis en su utilización. (Ordoñez \& Roda, 2016). Se puede pensar en implementar un nuevo material sintético como alternativa de aplicación en el adobe, como es el polietileno tereftalato (PET) (Industrias JQ, 2019). Las botellas plásticas de polietileno de tereftalato (PET), constituyen un serio problema ecológico, higiénico, sanitario, político social y económico, pues, el costo de recolección, transporte y eliminación es cada vez más caro; desde el punto de vista estructural se está desaprovechando su potencial de uso como materia prima para la construcción. (Industria Nacional de Vías).

Es preciso aclarar los estudios realizados por el arquitecto "Soto Zumba Marco Leonardo" (Soto, 2012), donde identifica que el adobe al mezclarlo con otro material (PET), proporciona una aislación adecuada. Así mismo el estudio propuesto por la doctora Rosana Gaggino, establece diferentes sistemas de ladrillos y placas prefabricadas con plásticos reciclados aptos para la autoconstrucción, donde el PET funciona como un buen aislante térmico y acústico. (Instituto JQ, 2019). Los estudios continúan y se elaboran ladrillos y placas con plásticos reciclados aptos para la construcción, llevado a cabo en el CEVE. Donde la investigación ha logrado desarrollar componentes de construcción livianos, de buena aislación térmica, y resistencia mecánica suficiente para cumplir la función de cerramiento lateral de viviendas. (Gaggino, 2008)

Para la investigación se propuso realizar bloques de adobe en base a 4 combinaciones, el suelo más un porcentaje de PET triturado adicional a la composición del bloque que va en orden del $0 \%, 5 \%, 10 \%$ y $15 \%$ en función al volumen de la muestra. Los ensayos para determinar las características físicas del suelo se lo hacen en base a los parámetros descritos en las normas INEN 690 (Instituto Ecuatoriano de Normalización, 1982) para contenido de humedad, INEN 696 (Instituto Ecuatoriano de Normalización, 1911) para análisis granulométrico, INEN 691 (Instituto Ecuatoriano de Normalización, 1982) para límite líquido y la INEN 692 (Instituto Ecuatoriano de Normalización, 1982) para límite plástico, mientras que su evaluación de calidad y elaboración de los bloques de adobe, se lo hace con las condiciones descritas en la norma peruana E080 (Ministerio de Vivienda Construcción Y Saneamiento, 2017). Para determinar los parámetros de variación de temperatura y acondicionamiento acústico se lo hace mediante la construcción de una cámara hermética, estos rigiéndose a las normas $\mathrm{NCH} 849$ (Instituto Nacional de Normalización, 2008) y NCH 2865 (Instituto Nacional de Normalización, 2005) respectivamente para térmico y acústico. Los valores obtenidos se los comparó con la normativa vigente de la NEC-11-CAP 13 [11] Eficiencia Energética, donde proporcionan datos de acondicionamiento acústico y temperatura 
óptima de confort térmico. Se evalúa además la resistencia a compresión de las muestras elaboradas. La norma (Ministerio de Vivienda Construcción y Saneamiento, 2017) establece ya valores de resistencia mínima para elementos de adobe, donde se especifica que debe ser mínimo 1MPA.

Se tiene valores de temperatura que establecen un ambiente óptimo de confort, como el descrito por la Norma Ecuatoria de la Construcción en el capítulo de eficiencia energética, (Norma Ecuatoriana de la Construcción NEC-2011, 2011) donde precisa que el valor debe estar entre 18 y $26^{\circ} \mathrm{C}$. De igual manera se ha establecido que para mantener un nivel de confort acústico en el diseño y la construcción de una edificación se debe considerar dos parámetros: Aislamiento acústico y acondicionamiento acústico, que se vincula a la comodidad frente a los ruidos. La norma ecuatoriana permite valores máximos de ruido según el tipo de instalación, lo cual establece que, para actividades de vivienda, estudio, dormitorios, bibliotecas, hoteles el nivel de presión sonora es de $50 \mathrm{dBA}$, mientras que para aulas de estudio es de $55 \mathrm{dBA}$. Para lo que se refiere a aislamiento acústico la norma NCh 352 (Instituto Nacional de Normalización, 2000), brinda valores convenientes de aislación para materiales en viviendas, la cual identifica que para un espacio receptor como dormitorios y el emisor siendo el exterior, tiene un valor de aislación mínima 40 dBA.

\section{Metodologia.}

Materiales: El material utilizado para la elaboración de mampuestos de adobe predomina en los sectores rurales de la provincia de Tungurahua. Fue extraído de dos lugares, de la parroquia de Pilahuin, y de la parroquia Pilisurco.

\section{Análisis del suelo}

a) Pruebas de campo: Se realiza dos ensayos de campo el primero es la prueba "Cinta de Barro" y la segunda prueba "Presencia de arcilla". (Ministerio de Vivienda Construcción y Saneamiento, 2017).

b) Proceso de hidratación del suelo: Se cierne el material antes de preparar el barro y luego someterla a un proceso de hidratación por lo menos 48 horas. (Ministerio de Vivienda Construcción y Saneamiento, 2017)

c) Bloques de prueba: Antes de implementar otros materiales al suelo, se elaboran bloques de adobes y determinar la cantidad de arcilla es la adecuada en el suelo, (Ministerio de Vivienda Construcción y Saneamiento, 2017). Si los bloques al secarse presentan desmenuzamiento, el suelo tiene mucha arena y deberá 
adicionarse arcilla, y si los bloques se fisuran al secarse, el suelo presenta mucha arcilla y deberá agregarse arena. (Blondet et al, 2010)

\section{d) Pruebas de laboratorio}

Contenido de humedad: Se determina mediante la relación de la cantidad de agua presente en la muestra de suelo entre la masa de suelo en estado seco, expresado en porcentaje. Se realiza este ensayo según la norma NTE INEN 690 "Determinación del contenido de agua, método de secado al horno.

Granulometría: Se determina el rango de partículas presentes en el suelo, expresado como un porcentaje del peso seco total. Generalmente se usa el análisis por tamizado aplicando la norma NTE INEN 696 "Análisis granulométrico en los áridos fino y grueso".

Límites de plasticidad o Límites de Atterberg: Se determina los estados de consistencia del suelo mediante el ensayo con el equipo copa Casagrande.

Límite líquido (LL): Es una frontera entre el estado semilíquido y plástico. El contenido de humedad del suelo debe expresarse como el porcentaje del agua, en relación con el peso de la muestra secada al horno. El ensayo se determina en base a la norma NTE INEN 691 "Mecánica de suelos. Determinación del límite líquido método de casa grande".

Límite plástico (LP): Es la frontera entre el estado plástico y semisólido. Este ensayo de laboratorio se rige a la norma NTE INEN 692 "Determinación del límite plástico".

Índice plástico (IP): Se determina el índice plástico como la diferencia numérica entre su límite Líquido y Límite Plástico. Este valor representa el porcentaje de humedad que deben tener las arcillas para conservarse en estado plástico.

e) Clasificación del suelo en base a la AASHTO: La asociación Americana de Carreteras Estatales y Transporte Oficial, AASHTO M - 145 realiza una clasificación del suelo, busca principalmente juzgar la aceptabilidad de un suelo basándose en la medición numérica de la calidad del suelo. La clasificación se basa en buscar en la figura 2 la primera columna de la izquierda a derecha que haga que todos los requisitos en el suelo encajen dentro de los rangos definidos, de acuerdo a la granulometría por tamizado y a los límites de consistencia.

\section{Adición del plástico triturado PET}

Para su implementación en los mampuestos, debido a que es un material que tiene mucho volumen y poca densidad, ocupa mucho espacio y pesa poco, la relación al volumen necesario para la muestra de adobe, fue el porcentaje de PET en 5\%,10\% y $15 \%$. 


\section{Elaboración de mampuestos}

Formas y dimensiones del adobe: El bloque de adobe rectangular debe tener un largo igual a dos veces su ancho, puede ser de planta cuadrada o rectangular y en el caso de encuentros, de formas especiales, pueden tener ángulos diferentes de $90^{\circ}$, no debe sobrepasar los $0.40 \mathrm{~m}$. de lado, por razones de peso, y la altura del bloque de adobe debe medir entre $0.08 \mathrm{~m}$ y $0.12 \mathrm{~m}$. (Ministerio de Vivienda Construcción y Saneamiento, 2017).

Secado, curado y resistencia de mampuestos: Debe ser lento, para lo cual se realiza sobre tendales protegidos del sol y del viento. Sobre el tendal (que no debe ser de pasto, ni empedrado, ni de cemento) se debe espolvorear arena fina para eliminar restricciones durante el encogimiento de secado. Se deja secar por 10 días en un ambiente cubierto, se voltean para tener un secado uniforme hasta alcanzar un período de 28 días desde su fabricación. Para el ensayo de resistencia se selecciona tres muestras de adobe que no contengan fisuras ni deformaciones, colocar dos en la base con una separación de 30 $\mathrm{cm}$ y sobre ellos el tercer adobe, los adobes deberán soportar el peso de una persona promedio por un minuto. (Blondet et al, 2010).

Se elaboraron 200 bloques de $40 \mathrm{~cm}$ de largo, $20 \mathrm{~cm}$ de alto y $10 \mathrm{~cm}$ de espesor, que fueron ensayados, con un volumen ocupado que corresponde al $100 \%$ de una muestra de adobe, posterior a eso se determinó la cantidad representada en volumen en porcentaje de PET propuesto, 5\%, 10\%, y 15\%. Conformando la dosificación utilizada.

\section{Cámara hermética de ensayo}

Consta de estructura de metal de $1.5 \mathrm{~mm}$ de espesor y revestimiento con planchas de yeso cartón de $10 \mathrm{~mm}$, con aislación entregada y lana de vidrio entre placas.

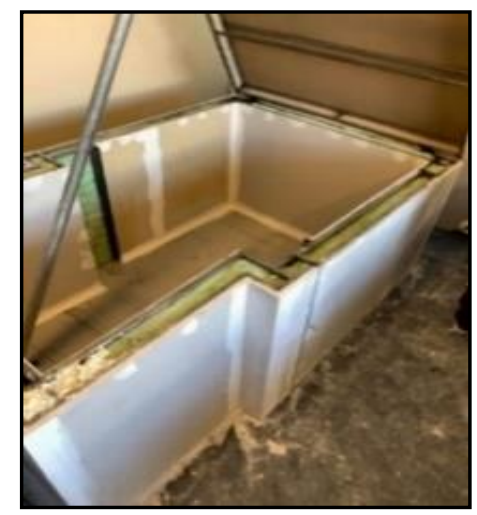

Figura 1. Fabricación de la cámara hermética

Fuente: Elaboración propia. 
La tapa es removible, con un sistema de aislamiento para el interior de la cámara. La pared por ensañarse se coloca de manera vertical en su interior.

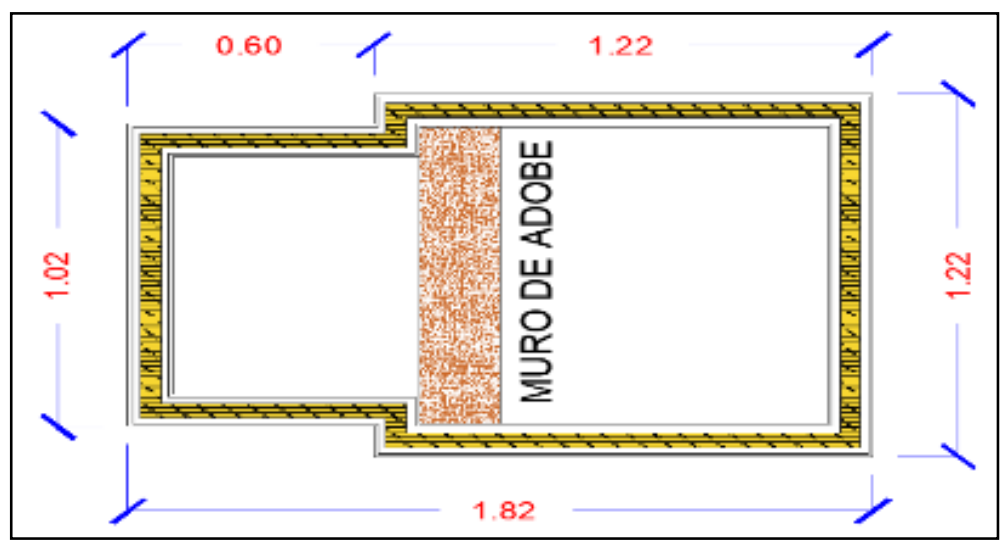

Figura 2. Disposición del muro de adobe

Fuente: Elaboración propia.

\section{Ensayo térmico}

Para la realización de este ensayo, se lo hace a través de normas chilenas, las cuales son: NCh. 851 of 1983 y NCh. 849 of 1987 Aislación Térmica. (Instituto Nacional de Normalización, 2008), con el cual se establecen parámetros comparativos entre las caras y ambientes de la pared que se someten a diferencia de temperaturas, por medio los sensores del equipo XPLORER GLX se obtienen los datos.

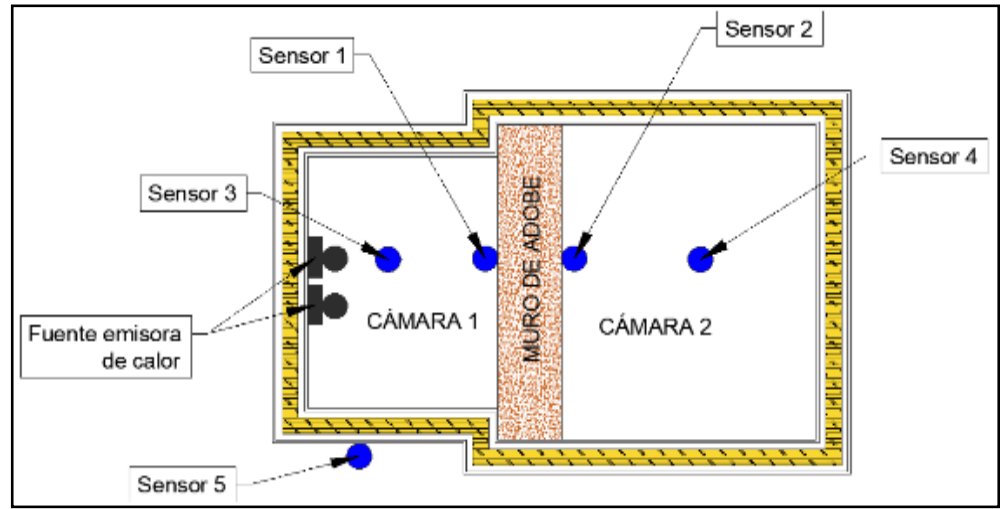

Figura 3 Disposición de sensores en la cámara hermética para ensayo de temperatura

Fuente: Elaboración propia. 
Sensor 1: mide la tempera de la cara 1

Sensor 2: mide la tempera de la cara 2

Sensor 3: mide la tempera del ambiente 1

Sensor 4: mide la tempera del ambiente 2

Sensor 5: mide la tempera exterior de laboratorio.

Con el equipo emisor de calor, que consta de 6 focos de 100 watts, se evalúan los muros de adobe obteniendo datos de temperatura en las caras 1 y 2 del muro y los ambientes 1 y 2 de la cámara, por 24 horas, durante un mes. En las tablas se ponen los promedios obtenidos.

Evaluación del nivel de aislación térmica Se evalúa el nivel de aislación térmica producida entre las caras del elemento y los ambientes separados, además de determinar el coeficiente de conductividad térmica, la cual verifica el grado de transferencia de calor, mediante la siguiente fórmula emitida por la norma NCh 851 (Instituto Nacional de Normalización, 2007).

$$
\lambda=\frac{d * P}{A(\Delta t)}
$$

Donde:

$\mathrm{d}=$ Espesor del elemento

$\mathrm{P}=$ Potencia

$\mathrm{A}=$ Área

$\Delta t=$ Diferencia de temperatura de las caras del elemento

Evaluación del nivel de temperatura óptima de confort térmico: La temperatura óptima de confort se evalúa en el ambiente 2 de la cámara de ensayo, Este valor es necesario para establecer, si el bloque de adobe presenta un acondicionamiento térmico adecuado, basándolo en lo descrito por la norma NEC 2011 capítulo de eficiencia energética (Instituto Nacional de Normalización, 2000), además de comprarlo con la bibliografía consultada.

\section{Ensayo acústico}

Se utiliza para medir la aislación acústica de elementos de la construcción pequeños, bajo condiciones de campo difuso. Esta norma entrega los resultados que se pueden utilizar para diseñar elementos de construcción con propiedades acústicas apropiadas. NCh. 2865 of 2004 Acústica (Instituto Nacional de Normalización, 2005). se somete pared una diferencia sonora entre los ambientes y mediante los sensores se tienen los datos. 


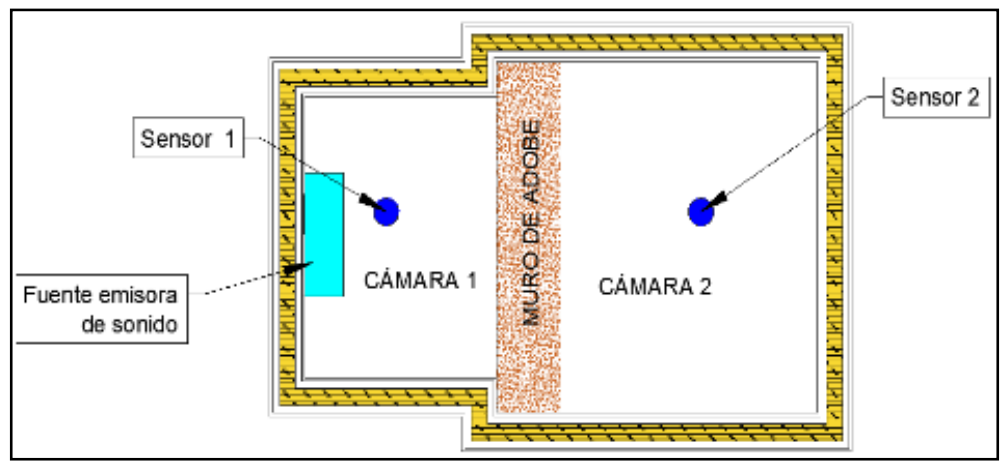

Figura 4. Disposición de la cámara hermética para ensayo de sonido Fuente: Elaboración propia.

Sensor 1: equipo (sonómetro) que mide la presión sonora en la cámara 1

Sensor 2: equipo (sonómetro) que mide la presión sonora en la cámara 2, luego de realizar la medición en la cámara 1.

Para los registros de presión sonora se tomará en un tiempo de 24 horas por un mes, registrando los valores máximos, mínimos, y promedios.

Determinación del nivel óptimo de confort acústico: Se considera dos parámetros el aislamiento y acondicionamiento acústicos.

Aislación acústica: Se determina en base a una diferencia entre el nivel de presión sonora de las caras del muro de adobe, se realiza una comparación de los datos obtenidos entre la bibliografía investigada y el nivel de aislación conveniente requerida en materiales entregado por la norma NCh 352. (Instituto Nacional de Normalización, 2000)

Acondicionamiento acústico: Se evalúa en función a los niveles máximos de ruido permitidos expresado en lo la norma NEC 2015 capítulo de eficiencia energética. (Instituto Nacional de Normalización, 2000) Al verificar si el material ensayado cumple con lo especificado anteriormente, se podrá determinar si el bloque de adobe propuesto es un elemento que brinda confort acústico.

\section{Resultados.}

Bloques de prueba: Se procedió a colocar $45 \%$ de cada suelo extraído de las zonas especificadas previamente y añadido un $10 \%$ de arena gruesa del sitio de estudio, dando como resultado que el suelo cumplen con la cantidad óptima de arcilla.

Tabla 1: Ensayos de campo realizados al suelo 


\begin{tabular}{|l|c|c|}
\multicolumn{1}{|c|}{ Ensayo } & \multicolumn{2}{c|}{ Verificación } \\
\hline Cinta de barro & Cumple (l=12cm) \\
\hline Resistencia seca & Cumple \\
\hline Contenido de humedad & \multicolumn{2}{c|}{$29.29 \%$} \\
\hline \multirow{3}{*}{ Granulometría } & Arena & $63 \%$ \\
\cline { 2 - 3 } & Limo & $15 \%$ \\
\cline { 2 - 3 } & Arcilla & $22 \%$ \\
\hline
\end{tabular}

Fuente: Elaboración propia.

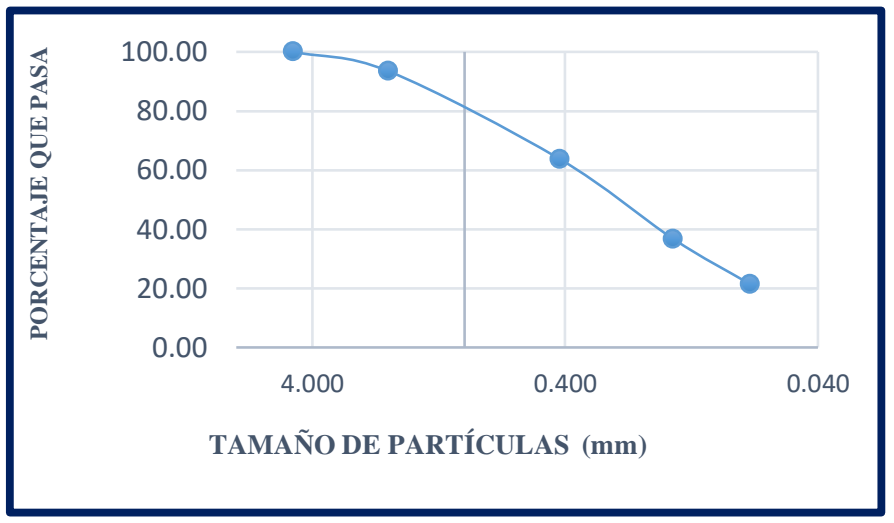

Figura 5 Curva granulométrica del suelo

Fuente: Elaboración propia.

Tabla 2: Límites de Atterberg

\begin{tabular}{|l|l|}
\hline Límite Líquido & $45.95 \%$ \\
\hline Límite Plástico & $33.13 \%$ \\
\hline Índice de plasticidad & $12.83 \%$ \\
\hline
\end{tabular}

Fuente: Elaboración propia.

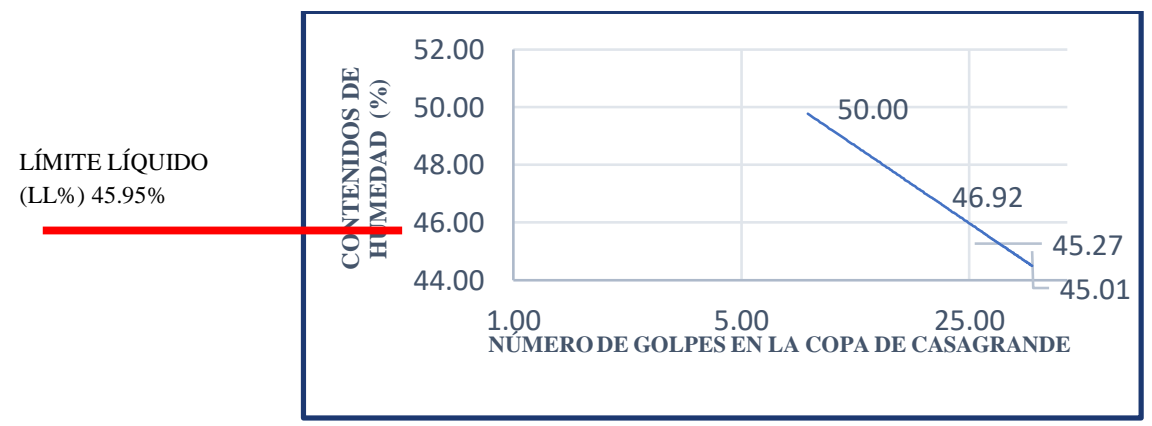

Figura 6. Curva granulométrica del suelo

Fuente: Elaboración propia. 


\section{Adición del plástico triturado PET}

Tabla 3. Dosificación de las muestras de bloques de adobe para ensayos.

\begin{tabular}{|c|c|c|c|c|c|c|}
\hline Ensayo & Nombre & Suelo & $\begin{array}{c}\text { Material } \\
\%\end{array}$ & $\begin{array}{c}\text { Agua } \\
\%\end{array}$ & $\begin{array}{c}\text { PET } \\
\%\end{array}$ & $\begin{array}{c}\text { \# de } \\
\text { muestras }\end{array}$ \\
\hline \multirow{4}{*}{$\begin{array}{l}\text { Térmico y } \\
\text { acústico }\end{array}$} & Adobe $+0 \%$ PET & Adobe & 100 & 12 & 0 & 16 \\
\hline & Adobe + 5\% PET & Adobe & 100 & 12 & 5 & 16 \\
\hline & Adobe $+10 \%$ PET & Adobe & 100 & 12 & 10 & 16 \\
\hline & Adobe $+15 \%$ PET & Adobe & 100 & 12 & 15 & 16 \\
\hline \multirow{4}{*}{ Compresión } & Adobe $+0 \%$ PET & Adobe & 100 & 12 & 0 & 6 \\
\hline & Adobe $+5 \%$ PET & Adobe & 100 & 12 & 5 & 6 \\
\hline & Adobe $+10 \%$ PET & Adobe & 100 & 12 & 10 & 6 \\
\hline & Adobe $+15 \%$ PET & Adobe & 100 & 12 & 15 & 6 \\
\hline
\end{tabular}

Fuente: Elaboración propia.

Tabla 4. Resumen de valores de temperatura en las caras 1 y 2 de los muros ensayados

\begin{tabular}{|c|c|c|c|c|c|}
\hline & Tipo & $\begin{array}{l}\text { T. máx } \\
{ }^{\circ} \mathrm{C}\end{array}$ & $\begin{array}{l}\text { T. mín } \\
{ }^{\circ} \mathrm{C}\end{array}$ & $\begin{array}{c}\text { T. Prom } \\
{ }^{\circ} \mathrm{C}\end{array}$ & $\begin{array}{c}\text { Min-Max } \\
{ }^{\circ} \mathrm{C}\end{array}$ \\
\hline \multirow{4}{*}{ CARA 1} & $\mathrm{~A}+0 \%$ PET & 33.54 & 14.49 & 25.89 & 19.04 \\
\hline & A. $+5 \%$ PET & 51.30 & 19.86 & 40.21 & 31.44 \\
\hline & A.+ $10 \%$ PET & 48.08 & 19.47 & 37.39 & 28.61 \\
\hline & A.+ $15 \%$ PET & 39.32 & 14.15 & 29.83 & 25.16 \\
\hline \multirow{4}{*}{ CARA 2} & A.+ $0 \%$ PET & 14.75 & 14.66 & 14.70 & 0.09 \\
\hline & A.+ 5\% PET & 14.80 & 14.33 & 14.58 & 0.47 \\
\hline & A. $+10 \%$ PET & 15.17 & 14.73 & 14.96 & 0.45 \\
\hline & A. $+15 \%$ PET & 14.29 & 14.18 & 14.24 & 0.12 \\
\hline
\end{tabular}

Fuente: Elaboración propia.

Tabla 5. Niveles de aislación y coeficiente de conductividad térmica de los bloques de adobe

\begin{tabular}{c|c|c|} 
Tipo & Aislación & $\boldsymbol{\lambda}$ \\
& ${ }^{\circ} \mathbf{C}$ & $\mathbf{w} / \mathbf{m}^{\circ} \mathbf{K}$. \\
\hline ADOBE + 0\% PET & 18.78 & 0.57 \\
\hline ADOBE + 5\% PET & 36.49 & 0.54 \\
\hline ADOBE + 10\% PET & 32.91 & 0.55 \\
\hline ADOBE + 15\% PET & 25.02 & 0.56 \\
\hline
\end{tabular}

Fuente: Elaboración propia. 


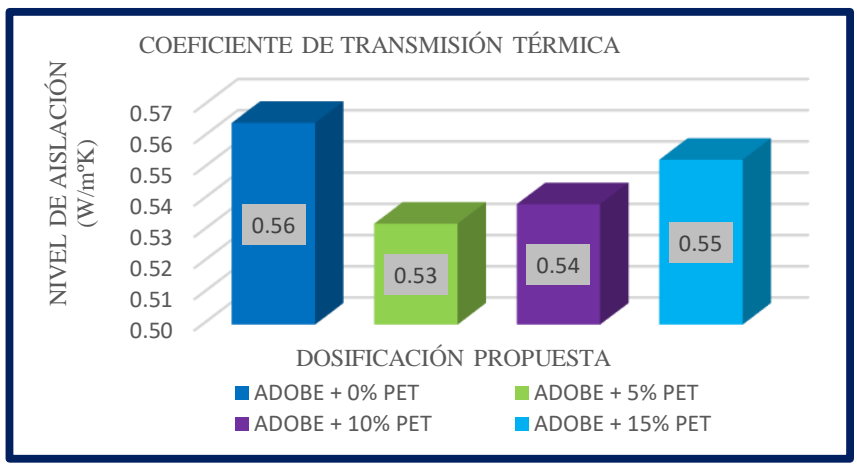

Figura 7. Coeficiente de transmisión térmica $\left(\mathrm{W} / \mathrm{m}^{\circ} \mathrm{K}\right)$ vs \% PET

Fuente: Elaboración propia.

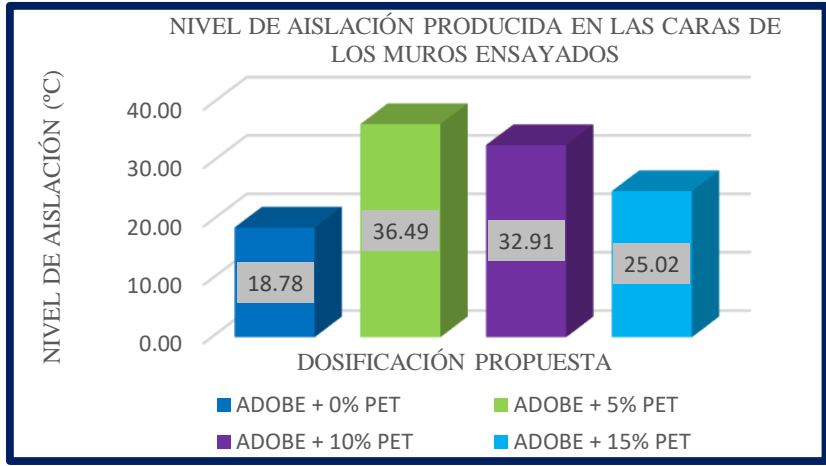

Figura 8. Nivel de aislación $\left({ }^{\circ} \mathrm{C}\right)$ en las caras del muro vs \% PET

Fuente: Elaboración propia.

Tabla 6. Resumen de valores de temperatura ambientes 1 y 2 de los muros ensayados

\begin{tabular}{|c|c|c|c|c|c|c|}
\hline \multirow{4}{*}{ AMB. } & Tipo & T. máx & T. mín & T. Prom & Min-Max & Ais. \\
\cline { 2 - 6 } $\mathbf{1}$ & A.+ 0\% PET & 46.63 & 13.94 & 35.69 & 32.69 & 30.62 \\
\cline { 2 - 6 } & A.+ 5\% PET & 54.58 & 18.28 & 42.50 & 36.29 & 38.59 \\
\cline { 2 - 6 } & A.+ 10\% PET & 53.55 & 19.39 & 42.26 & 34.15 & 37.47 \\
\cline { 2 - 6 } & A.+ 15\% PET & 51.06 & 14.00 & 38.67 & 37.06 & 35.33 \\
\hline \multirow{3}{*}{$\begin{array}{c}\text { AMB. } \\
\mathbf{2}\end{array}$} & A.+ 0\% PET & 16.02 & 14.39 & 15.80 & 1.63 & \\
\cline { 2 - 6 } & A.+ 5\% PET & 15.99 & 13.42 & 15.56 & 2.57 & \\
\cline { 2 - 6 } & A.+ 10\% PET & 16.07 & 13.67 & 15.66 & 2.40 & \\
\cline { 2 - 6 } & A.+ 15\% PET & 15.73 & 13.77 & 15.39 & 1.95 & \\
\hline
\end{tabular}

Fuente: Elaboración propia. 


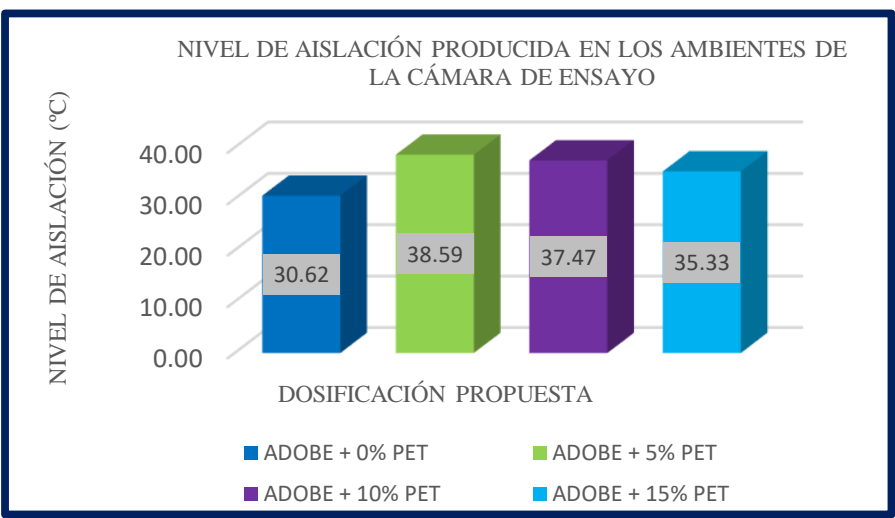

Figura 9. Nivel de aislación $\left({ }^{\circ} \mathrm{C}\right)$ en los ambientes vs \% PET

Fuente: Elaboración propia.

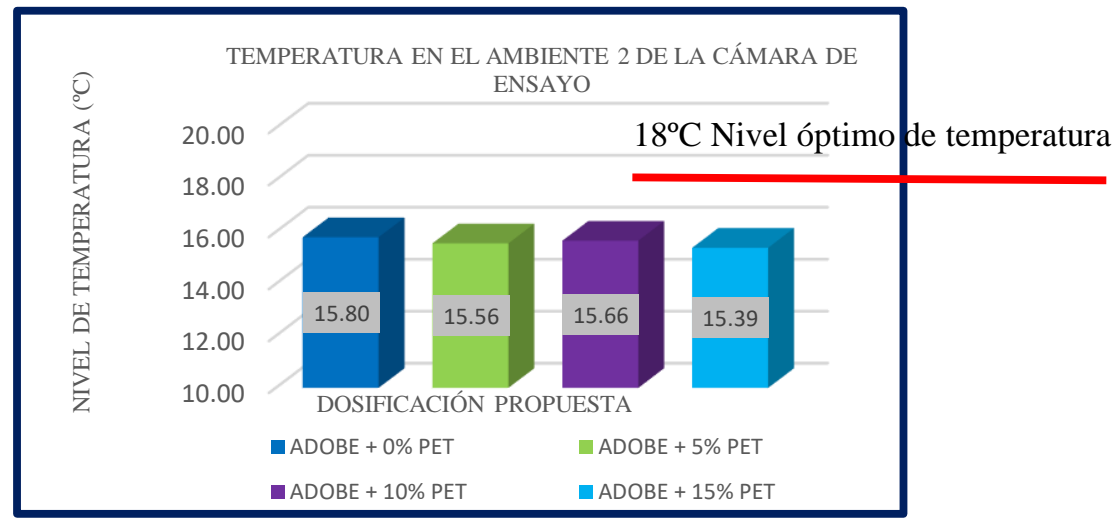

Figura 10. Nivel de Temperatura de $\left({ }^{\circ} \mathrm{C}\right)$ en el ambiente 2 vs $\%$ PET

Fuente: Elaboración propia.

\section{Comparación de resultados de ensayo térmico}

Tabla 7. Comparación de resultados de ensayo térmico.

\begin{tabular}{|l|l|l|l|l|l|}
\hline Comparación & Unidad & $\begin{array}{c}\text { Adobe } \\
+\end{array}$ & $\begin{array}{c}\text { Adobe } \\
+\end{array}$ & $\begin{array}{l}\text { Adobe } \\
+\end{array}$ & $\begin{array}{l}\text { Adobe } \\
+\end{array}$ \\
\hline $\begin{array}{l}\text { Coeficiente de conductividad } \\
\text { térmica aparente de las } \\
\text { combinaciones propuestas en } \\
\text { esta investigación. }\end{array}$ & $\mathrm{w} / \mathrm{m} \mathrm{K}$ & 0.57 & 0.54 & 0.55 & 0.56 \\
\hline $\begin{array}{l}\text { Coeficiente de conductividad } \\
\text { térmica aparente. }\end{array}$ & $\mathrm{w} / \mathrm{m} \mathrm{K}$ & 0.90 & 15\% PET \\
\hline $\begin{array}{l}\text { Coeficiente de conductividad } \\
\text { térmica aparente. }\end{array}$ & $\mathrm{w} / \mathrm{m} \mathrm{K}$ & 0.15 & \\
\hline
\end{tabular}

Fuente: Elaboración propia. 
Tabla 8. Resumen de valores de presión sonora en los ambientes 1 y 2 de los muros ensayados

\begin{tabular}{|c|c|c|c|c|c|c|c|c|}
\hline & \multicolumn{2}{|c|}{ 0\% PET } & \multicolumn{2}{|c|}{ 5\% PET } & \multicolumn{2}{|c|}{$10 \%$ PET } & \multicolumn{2}{|c|}{$15 \%$ PET } \\
\hline & $\mathbf{E}$ & $\mathbf{R}$ & $\mathbf{E}$ & $\mathbf{R}$ & $\mathbf{E}$ & $\mathbf{R}$ & $\mathbf{E}$ & $\mathbf{R}$ \\
\hline & $\mathrm{dBA}$ & $\mathrm{dBA}$ & $\mathrm{dBA}$ & $\mathrm{dBA}$ & $\mathrm{dBA}$ & dBA & $\mathrm{dBA}$ & $\mathrm{dBA}$ \\
\hline Máx. & 85.00 & 48.00 & 85.50 & 45.30 & 84.50 & 38.80 & 86.10 & 41.70 \\
\hline Mín. & 82.00 & 45.10 & 82.40 & 39.40 & 80.70 & 37.90 & 82.80 & 38.00 \\
\hline Promedio & 83.65 & 46.32 & 84.01 & 43.49 & 81.91 & 38.34 & 83.68 & 39.79 \\
\hline Max-Min & 3.00 & 2.90 & 3.10 & 5.90 & 3.80 & 0.90 & 3.30 & 3.70 \\
\hline Aislamiento & \multicolumn{2}{|c|}{$\mathbf{3 7 . 3 3}$} & \multicolumn{2}{|c|}{40.52} & \multicolumn{2}{|c|}{43.57} & \multicolumn{2}{|c|}{43.89} \\
\hline
\end{tabular}

Fuente: Elaboración propia.

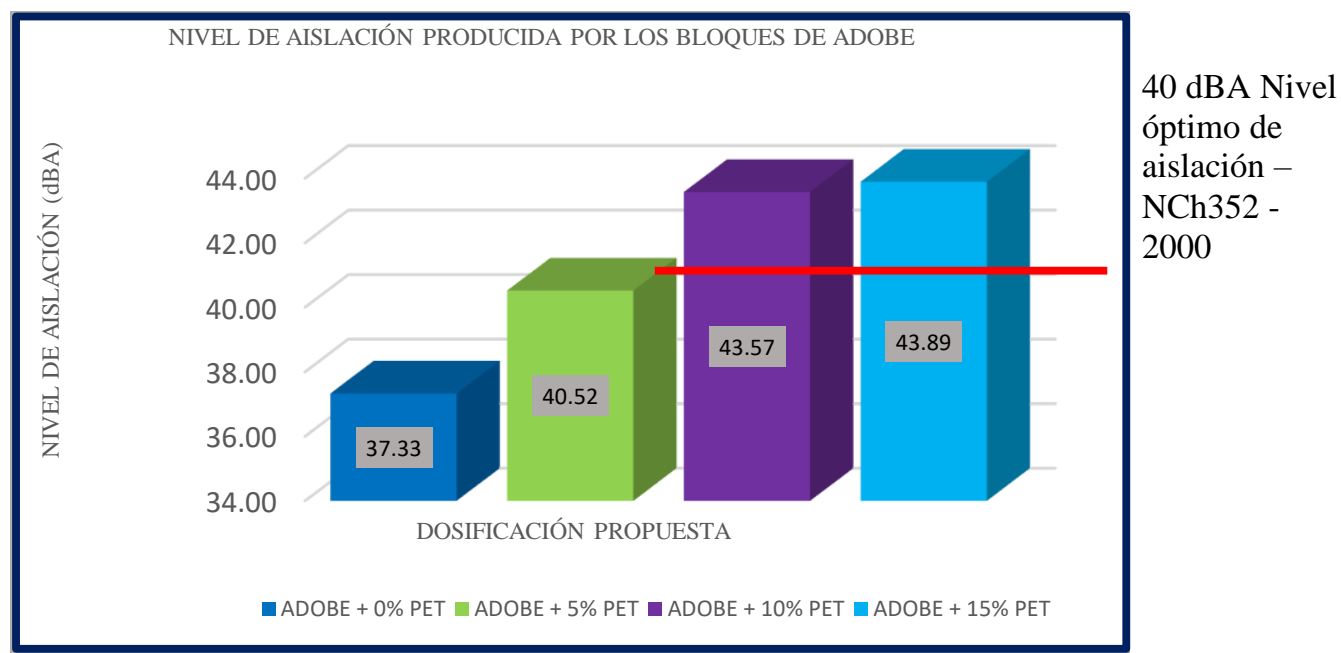

Figura 11. Nivel de aislación (dBA) de bloques de adobe ensayados

Fuente: Elaboración propia.

Tabla 9. Comparación de resultados de ensayo acústico en función de las resultados obtenidos y bibliografía consultada.

\begin{tabular}{|l|c|c|c|c|c|}
\multicolumn{1}{|c|}{ Comparación } & Unidad & $\begin{array}{c}\text { Adobe } \\
+ \\
\text { 0\% PET }\end{array}$ & $\begin{array}{c}\text { Adobe } \\
+\end{array}$ & $\begin{array}{c}\text { Adobe } \\
\text { 5\% PET }\end{array}$ & $\begin{array}{c}\text { Adobe } \\
+\end{array}$ \\
\hline $\begin{array}{l}\text { Aislación producida en } \\
\text { los ambientes de la } \\
\text { cámara de ensayo }\end{array}$ & dBA & 37.33 & 40.52 & 43.57 & 43.89 \\
\hline $\begin{array}{l}\text { Aislación ladrillo con } \\
\text { PET reciclado e =12 cm }\end{array}$ & $\mathrm{dBA}$ & \multicolumn{5}{|c|}{20} \\
\hline Umbral de audición & $\mathrm{dBA}$ & \multicolumn{5}{|c|}{40} \\
\hline
\end{tabular}

Fuente: Elaboración propia. 
Se puede establecer, que el nivel de aislación va en aumento en función al incremento de PET, además de establecer que sobrepasa el nivel de aislación mínima que debe cumplir las construcciones habitacionales expuesta por la norma NCh 352 en un 8.86\%. Además, las tres combinaciones propuestas con material PET están por encima del nivel requerido de aislación mínima que es 40 dBA.

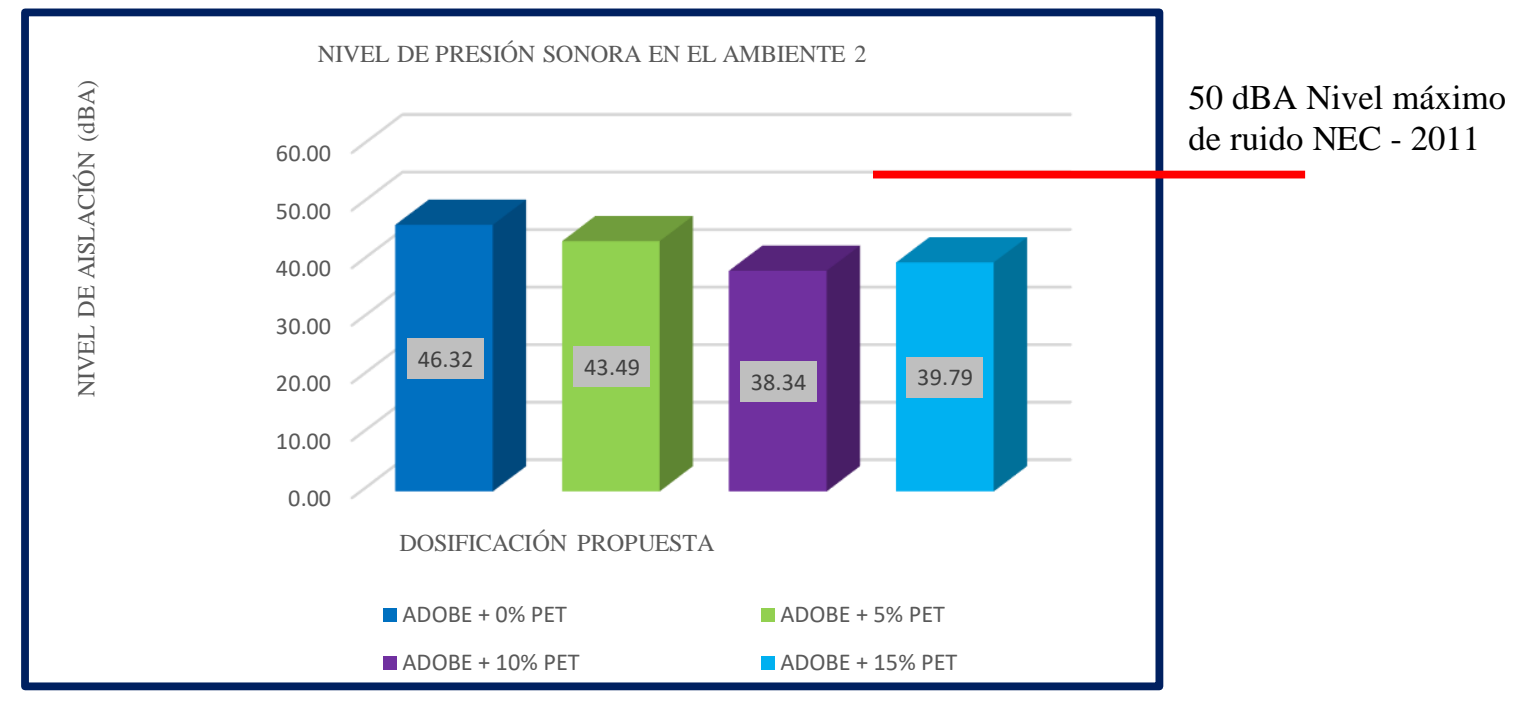

Figura 21. Nivel de aislación (dBA) de bloques de adobe ensayados

Fuente: Elaboración propia.

\section{Conclusiones.}

- Realizando el análisis de temperatura de las combinaciones propuestas, se tiene que, con el bloque de adobe más 5\% de material PET existe un mayor valor de aislación térmica, además al ser comparado con el bloque artesanal presenta el menor valor de coeficiente de transmisión térmica con un 5.53\% inferior al mismo adobe artesanal. Sabiendo que mientras más bajo el valor del coeficiente de transmisión térmica el material funciona como un mejor aislante de temperatura.

- El nivel de acondicionamiento acústico está en un rango óptimo, puesto que el nivel de presión sonora es inferior al máximo permitido por la norma NEC 2011 en su capítulo de eficiencia energética, donde se especifica que, para actividades de vivienda, estudio, dormitorios, bibliotecas, hoteles, lugares de estar, se tiene un nivel de sonido máximo de $50 \mathrm{dBA}$, y para aulas de estudio de 55 dBA.

- Las cuatro combinaciones propuestas en la investigación no cumplen con la temperatura óptima de confort térmico en relación a la temperatura descrita según la NEC 2011 en su capítulo de eficiencia energética, el cual se define en un rango de $18-20{ }^{\circ} \mathrm{C}$ [14], esto se puede atribuir a su composición, sin 
embargo, este material funciona como un buen aislante térmico, por lo que se sugiere que la implementación de estos mampuestos sea en zonas cálidas.

- Mediante el análisis de presión sonora de las diferentes combinaciones propuestas, se tiene que el mayor nivel de aislación acústica, obtenido mediante la diferencia entre el ambiente emisor y el ambiente receptor de la cámara de ensayo, se produce con el bloque de adobe más un 15\% de material PET. Además, teniendo en cuenta el valor mínimo de aislación que debe cumplir las construcciones habitacionales expuesta por la norma NCh 352 es de $40 \mathrm{~dB}$, por lo que se verifica que es superior a este valor en un $8.86 \%$, siendo óptimo para mantener el confort acústico en viviendas unifamiliares.

- Evaluando el nivel de plástico PET triturado, la temperatura y el sonido, se determina que el porcentaje óptimo de PET es el 5\% en relación al volumen de las muestras de adobe, mantiene una aislación térmica adecuada, un nivel de acondicionamiento y aislación acústica óptima por lo que presenta un confort adecuado, además de mantener una resistencia a compresión superior al emitido por la normativa.

\section{Referencias bibliográficas.}

2786, N. (2003). Acustica. Medición de Aislación acústica en construcciones y elementos de construcción. Mediciones en Laboratorio de la Aislación acustica aerea de elementos de construcción. Santiago de Chile : Norma Chilena Oficial.

3066., I. (2016). Bloques de hormigón. requisitos y métodos de ensayo. Quito: Norma Técnica Ecuatoriana.

849, N. (1987). Aislación Térmica. Transmisión Térmica. Terminología, Magnitudes, Unidades y Símbolos. Santiago de Chile : Norma Chilena Oficial .

ANDREA, V. G. (2018). Refuerzo estructural con PET reutilizado aplicación en Adobe. Madrid - España: E.T.S. Arquitectura (UPM).

ANTONIO, I. V. (2018). Guía de construcción para solucionar problemas de aislamiento acústico en paredes de bloque hueco de hormigón usando aserrín pulverizado . Quito - Ecuador.

Apuntes de Geotecnia con énfasis en laderas. (18 de noviembre de 2010). (geotecniasor.blogspot.com) Recuperado el 21 de Marzo de 2019, de http://geotecniasor.blogspot.com/2010/11/consistencia-del-suelo-limites-de.html

Bernal, M., \& Palacio, N. (2018). Correlacion entre las propiedades de los bloques ecologicos fabricados con los componentes del Tetra pak reciclado y bloques convencionales. Quito: Tesis de Grado. 
BLONDET, M. (2010). Manual de construcción con adobe reforzado con geomallas De viviendas de bajo costo saludables y seguras. Perú.

Bravo, E., Poveda, A., \& Ubico, D. (2007). Manual de Construccion con Bloques de Concreto. San Jose: ICCYC.

CARLA VALENTINA RODAS LEÓN, J. F. (2016). Desarrollo tecnológico, investigativo y experimental de ecobloques de hormigón en base a vidrio y polietileno de tereftalato (PET) reciclado como alternativa sustentable al bloque tradicional . Cuenca - Ecuador .

CHILE, I. N. (2000). NCh 352. Aislación Acústica - Construcciones de uso habitacional - Requisitos Mínimos y ensayo. Chile.

CHILE, I. N. (2005). NCh 2865: Acústica - Medición de aislación acústica en construcciones y elementos de construcción - Medición en laboratorio de aislación acústica aérea de elementos de construcción pequeños . Chile.

CHILE, I. N. (2007). NCh 853: Acondicionamiento térmico - Envolvente térmica de edificios - Cálculo de resistencias y transmitancias térmicas. Chile.

CHILE, I. N. (2008). NCh 851: Aislación térmica - Determinación de propiedades de transmisión térmica en estado estacionario y propiedades relacionadas - Cámara térmica calibrada y de guarda. Chile.

Conrado, M., \& Rojas, A. (2012). Diseño de hormigones con fibras de polipropileno para resistencias a la compresion de 21 y $28 \mathrm{MPa}$ con agregados de la cantera de Guayllabamba. Quito: Tesis de Grado.

CONSTRUCCIÓN, N. E. (2011). Capítulo 13 - Eficiencia Energética en la Construcción en Ecuador. Quito - Ecuador .

CONSTRUCCION, N. E. (2011). EFICIENCIA ENERGÉTICA EN LA CONSTRUCCION EN ECUADOR. Quito: MIDUVI.

EVANS, S. D. (2006). Construcción con tierra. Buenos Aires - Argentina: Centro de Investigación Habitat y Energía, CIHE, SI, FADU, UBA.

Flores, V., Torres, R., Vallejos, R., \& Flores, M. (2014). Mezclas de cemento y agregados de plástico para la construccion de viviendas ecológicas. Chuquisaca: ECORFAN.

GAGGINO, R. (2008). Ladrillos y placas prefabricadas con plásticos reciclados aptos para la construcción. Revista Invi N63, 23(137), 27. 
Gavilán, \& Juan. (2014). Evaluacion termica y acustica de paneles fabricados con ladrillos ecologicos. Valparaiso: Tesis de Grado.

Gomá, F. (1979). Cemento Portland y otros Aglomerantes. Barcelona: Editores Tecnicos Asociados S.A.

hgz, E. (23 de 03 de 2011). Ingeniro Civil. Recuperado el 25 de 04 de 2019, de http://uningenierocivil.blogspot.com/2011/03/limites-de-atterberg-indicede.html

INEN. (2016). BLOQUES DE HORMIGÓN. REQUISITOS Y MÉTODOS DE ENSAYO. Quito: Norma Técnica Ecuatoriana.

INSTRUMENTS, G. A. (s.f.). SHIMADZU: Excellense in Science. Recuperado el 26 de Abril de 2019, de https://www.shimadzu.com/an/test/universal/concreto.html

JIMÉNEZ, A. D. (2015). Determinación de la resistencia a compresión y la durabilidad del adobe prensado a máquina para la construcción de infraestructura agropecuaria rural en la parroquia Chuquiribamba. Loja - Ecuador .

JQ, I. (20 de Julio de 2016). Plásticos de Ingeniería . Recuperado el 25 de Abril de 2019, de http://www.jq.com.ar/info-pet.html

KAREN TATIANA ARTEAGA MEDINA, O. H. (2011). Bloque de tierra comprimida como material constructivo. Universidad Pedagógica y Tecnológica de Colombia , 20(31), 55-68.

LEONARDO, S. Z. (2012). Materiales aislantes acústicos para muros . Loja - Ecuador

LILIANA TRONCOSO, S. V. (2008). Reforzamiento estructural de construcciones de adobe. Quito - Ecuador : Corporación nacional editorial.

LUIS, M. A. (2016). Optimización del uso de adobe sismo resistente, como mateerial constructivo para viviendas familiares de bajo costo . Guayaquil - Ecuador .

M. BLONDET, J. V. (2011). Construcción sismorresistente en tierra: la gran experiencia contemporánea de la Pontificia Universidad Católica del Perú. ROSE School, Instituto de Estudios Superiores de Pavía (Italia), 63(523), 10.

MARTÍNEZ, L. F. (2010). Manual de procedimientos analíticos: Laboratorio de física de suelos . México. 
MATERIALS, A. S. (1998). ASTM D-422-63: Método de análisis de las partículas de suelo.

MATERIALS, A. S. (2005). ASTM D4318: Los métodos estándar de ensayo para: Límite Líquido, Límite de plástico, y el índice de plasticidad de los suelos.

Medina, S. (2016). HORMIGÓN. Ambato: Universidad Técnica de Ambato.

MEJÍA, J. G. (2010). Estudio de la factibilidad para reciclar envases plásticos de polietileno tereftalato (PET), en la ciudad de Guayaquil. Guayaquil - Ecuador .

NCh 2786. (2003). Acustica. Medicion de aislacion acustica en construcciones y elementos de cosntruccion. Mediciones en laboratorio de la aislacion acustica aerea de elementos de construccion. Santiago de Chile: Norma Chilena Oficial.

NCh 849. (1987). Aislacion termica. Transmicion termica. Terminologia, magnitudes, unidades y simbolos. Santiago de Chile: Norma Chilena Oficial.

NCh 851. (2008). Aislacion termica. Determinacion de propiedades de transmicion termica en estado estacionario y propiedades relacionadas. Camara termica calibrada y de guarda. Santiago de Chile: Instituto Nacional de Normalizacion - INN.

NORMALIZACIÓN, I. E. (1982). NTE INEN 292: Mecánica de suelos. Determinanción del límite plástico. Quito - Ecuador.

NORMALIZACIÓN, I. E. (1982). NTE INEN 690: Mecánica de suelos: Determinanción del contenido de agua - Método del secado al horno. Quito Ecuador.

NORMALIZACIÓN, I. E. (1982). NTE INEN 691: Mecánica de suelos. Determinanción del límite líquido. Métod de Casa Grande. Quito Ecuador.

NORMALIZACIÓN, I. E. (2011). NTE INEN 696: Áridos. Análisis granulométrico en los áridos, fino y grueso . Quito - Ecuador.

NORMALIZACIÓN, I. E. (2013). NTE INEN 154: Mallas y tamices para ensayo. Requisitos. Quito - Ecuador.

NTE INEN 152. (2012). Cemento Portland. Requisitos. Quito: Norma Técnica Ecuatoriana.

OFFICIALS, A. A. (s.f.). AASHTO M154: Clasificación del suelo.

Paéz, C. (2014). Determinacion de la carga permamente debida al peso de mamposteria de bloque en edificaciones de vivienda. Quito: Tesis de Grado. 
PASCO. (2002). Manual de Instrucciones. Sensor de temperatura/ nivel de sonido/ luz. Roseville: PASCO scientific.

PASCO. (2006). Guia de usuario Xplorer GLX. Roseville: PASCO scientific.

REQUEMA, J. (s.f.). Ruido en el interior de las viviendas. Revista de acústica , 18(3), $52-54$.

RICARDO YAMASHIRO, A. S. (1981). Diseño sísmico de construcciones de adobe y bloque estabilizado - Primera parte. New México - US.

Rodas, C., \& Ordoñez, F. (2016). Desarrollo tecnologico, investigativo y experimental de ecobloques de hormigón en base a vidrio y polietileno de tereftalato (PET) reciclado, como alternativa sustentable al bloque tradicional. Cuenca: Tesis de grado.

Ruiz, M., Luzuriaga, H., \& Rodríguez, C. (2015). El Comportamiento del consumidor y la cultura de reciclaje de residuos sólidos: Caso Mancomunidad PatatePelileo. . Revista digital de Medio Ambiente "Ojeando la agenda" (36), 15.

SALAZAR, S. M. (2017). Estudio de la resistencia a compresión del adobe artesanal estabilizado con paja, estiércol, savia de penca de tuna, sangre de toro y análisis de su comportamiento sísmico usando un modelo a escala . Ambato - Ecuador

SANEAMIENTO, M. D. (2017). Norma E080. Diseño y construcción con tierra reforzada. Perú.

Santacruz, W., \& Velasteguí, D. (2018). Determinación De Dosificación Para Elaborar Bloques Huecos De Hormigón Que Cumplan Con La Actual Norma Inen 3066. Quito: Tesis de Grado.

SANTILLÁN, M. G. (2006). Del concepto de ruido urbano al de paisaje sonoro . Red de Revistas Científicas de América Latina, el Caribe, España y Portugal, 1(10), 39-52.

SILVA, J. P. (2014). Evaluación térmica y acústica de paneles fabricados con ladrillos ecológicos. Chile.

Smart, E. S. (s.f.). Acerca de nosotros: Express Shop Smart. Recuperado el 14 de Junio de 2019, de https://www.shopsmartexpress.com/item/B07GJ5654Z/instrument-compactprofessional-sound-level-meter-with-backlit-display-high-accuracymeasuring-30db-130db\# 
SUAREZ, J. D. (2015). Evaluación del tereftalato de polietileno (PET) como agregado en la elboración de mortero para ladrillos y concreto . Bogotá - Colombia .

Tarbuck, E., \& Lutgens, F. (2005). Ciencias de la Tierra. Una introduccion a la geologia fisica. Madrid: Pearson Educacion S.A.

UTEST. (s.f.). Secado, pesado y clasificación: Pesado muestras, Tamices.

VÍAS, I. N. (s.f.). I.N.V.E - 125 - 07: Determinanción del límite líquido de los suelos. Colombia.

Vidaud, E. (28 de Octubre de 2013). CONSTRUCCIÓN Y TECNOLOGÍA EN CONCRETO. Recuperado el 21 de Febrero de 2018, de http://www.revistacyt.com.mx/index.php/ingenieria/60-de-la-historia-delcemento

Y, A. M. (2007). Acústica: La ciencia del sonido . Medellín - Colombia.

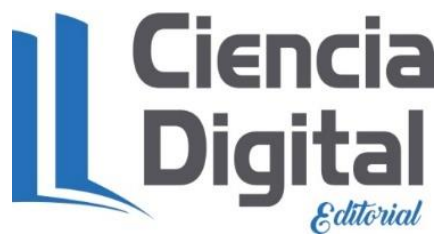




\section{PARA CITAR EL ARTÍCULO INDEXADO.}

Núñez Aldás, G. W., López Arboleda, A. G., Chérrez Gavilanes, D. S., \& Guevara Robalino, J. J. (2021). Adición de botellas plásticas pet en la elaboración de bloques de adobe para viviendas unifamiliares y su efecto en la variación de temperatura y acondicionamiento acústico en el cantón Ambato, provincia de Tungurahua. Ciencia Digital, 5(1), 197-128. https://doi.org/10.33262/cienciadigital.v5i1.1536

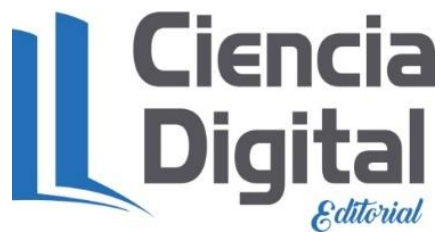

El artículo que se publica es de exclusiva responsabilidad de los autores y no necesariamente reflejan el pensamiento de la Revista Ciencia Digital.

El artículo queda en propiedad de la revista y, por tanto, su publicación parcial y/o total en otro medio tiene que ser autorizado por el director de la Revista Ciencia

\section{Digital.}
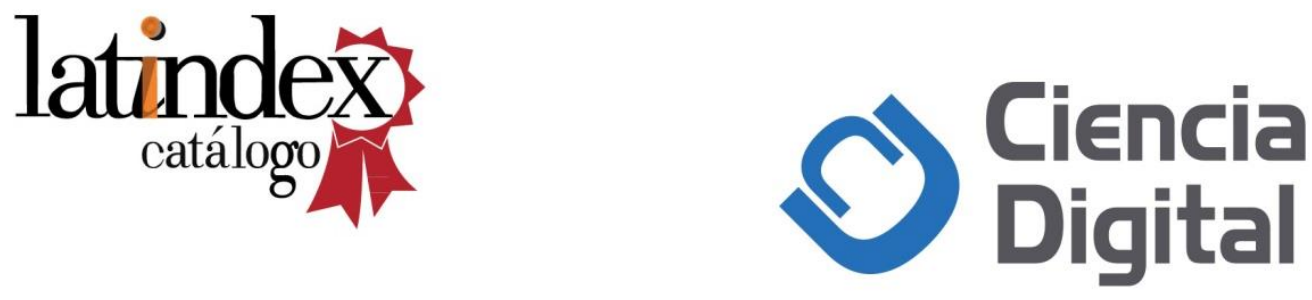